Sādhanā Vol. 40, Part 1, February 2015, pp. 89-106. (C) Indian Academy of Sciences

\title{
Experimental validation of waveform relaxation technique for power system controller testing
}

\author{
S P PANDA*,K A SALUNKHE and A M KULKARNI \\ Department of Electrical Engineering, Indian Institute of Technology, \\ Mumbai 400 076, India \\ e-mail: sakti@ee.iitb.ac.in; salunkheka@ee.iitb.ac.in; anil@ee.iitb.ac.in
}

MS received 7 February 2014; revised 26 July 2014; accepted 8 September 2014

\begin{abstract}
A Waveform Relaxation (WR) based iterative real-time playback scheme for controller testing was recently proposed in the literature along with proof-ofconcept simulations. This scheme can be a low-cost alternative to Hardware-in-Loop simulation, as it does not require a real-time simulator. To demonstrate practical feasibility of the WR scheme, this paper presents results of experiments with real-time implementation of controllers, iteratively interacting with simulated models of power apparatus via storage and real-time play-back. Two systems are considered: a HVDC controller tested with a detailed model of the converters, and a TCSC based damping controller tested with a low frequency model of a power system. The results are validated with those obtained using simulated models of the controllers. We also present results of an experiment in which the tested HVDC controller is used to control the scaled real-life HVDC apparatus, for which a simulated model was used during controller testing. Convergence and a good match between simulated and real-time implementation are obtained for the HVDC system. The experiments on the TCSC damping controller drawn our attention to a potential convergence problem which may arise due to iteration-dependent round-off noise.
\end{abstract}

Keywords. Power system controllers; controller testing; hardware in loop simulation; waveform relaxation method.

\section{Introduction}

Protection and control equipment have to be tested for an array of operating conditions and disturbances before their deployment in the field. For testing relay action which involves only one discrete triggering event, it is possible to use open-loop real-time playback of transients, which have been simulated in non-real-time (Kezunovic et al 2003)-see figure 1. Hardware In Loop (HIL) simulation (Kezunovic \& McKenna 1994) is a method of testing, wherein the power system is simulated using a real-time digital simulator and the controller hardware which is to be

${ }^{*}$ For correspondence 


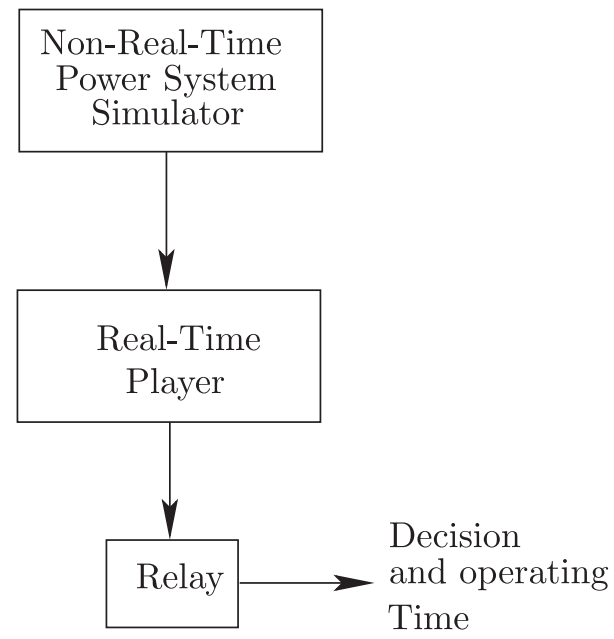

Figure 1. Real-time playback testing.

tested continuously exchanges feedback signals with the simulator - see figure 2. Even though real-time digital simulators are widely used, their cost increases as the size of the power system model increases. Moreover, if more than one controller, relay or measurement system, placed at geographically dispersed locations, is to be tested in situ with the associated communication system, then HIL simulation is difficult.

To overcome these difficulties of HIL based testing, a Waveform-Relaxation (WR) (White et al 1985) based iterative and real-time playback was proposed in (Kulkarni et al 2010). WR method was originally proposed as a method of parallelizing the numerical integration of very

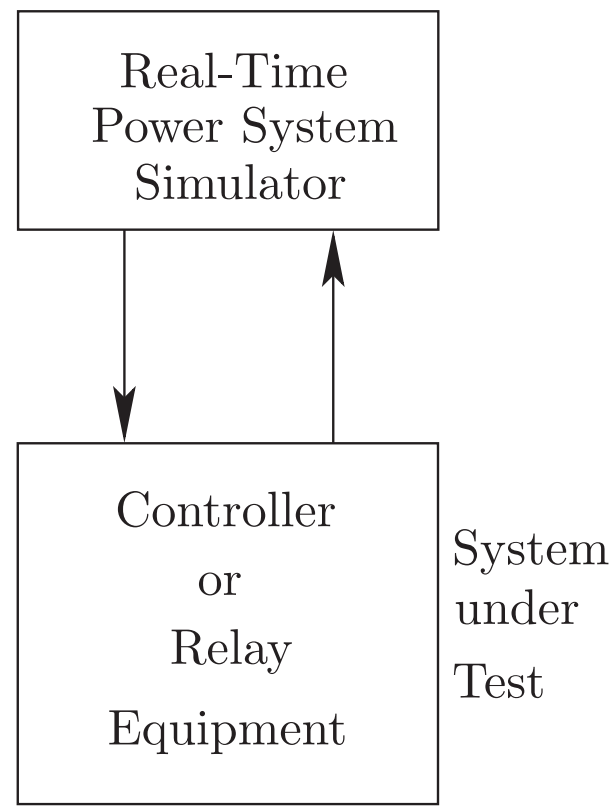

Figure 2. HIL testing. 
large systems (e.g., VLSI) (Lelarasmee et al 1982) and (White \& Sangiovanni-Vintocelli 1987). It was also used for parallelizing transient stability simulations of large power systems (Spong et al 1987). However, the issues in the application of WR for controller testing are distinct from the earlier applications (Kulkarni et al 2010).

The WR based iterative real-time playback scheme for testing of a single controller is shown in figure 3. The output of the controller under test at the $(k-1)^{t h}$ WR iteration, i.e., the time stamped waveform $\mathrm{y}_{\mathrm{c}}^{\mathrm{k}-1}(t)$, is fed as input to the non-real-time power system simulator. The power system is simulated (not in real-time) using this input and the simulated waveforms are stored along with their time-stamps. The stored waveforms are then played back in real-time to the controller again to obtain the controller response for the new iteration, i.e., $y_{c}^{k}(t)$. This response is stored and used again by the non-real-time simulator in the next iteration. This process is repeated till convergence is attained. When convergence is attained, say at the $l^{\text {th }}$ iteration, then it is equivalent to closing the controller loop in figure 3 , since $y_{c}^{l-1}(t) \approx y^{l}(t)$. In other words, if the WR iteration converges, then the converged response of the tested equipment is the same as the HIL response.

Since the method is iterative, it is much slower than HIL simulation. Besides, there are overheads due to storage, re-starting of simulations and controller initialization at each iteration. While eventual convergence has been attained in many complex power system examples simulated by the authors, convergence may be extremely slow in cases where power electronic switchings are modelled. Nonetheless, since the method does not require real-time simulation, it is much cheaper and flexible as compared to HIL simulation, and this motivates us to investigate this further.

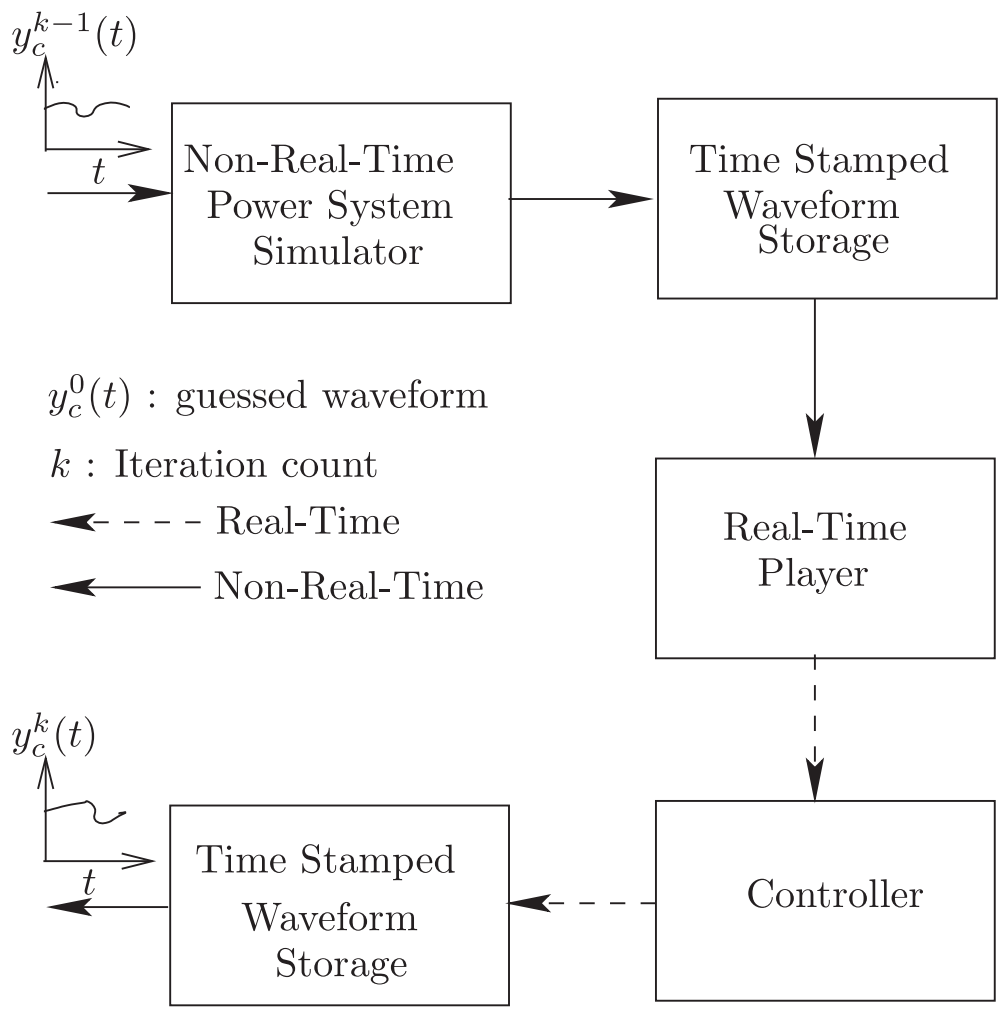

Figure 3. Waveform relaxation based iterative real-time playback. 
Table 1. Glossary of acronyms.

\begin{tabular}{ll}
\hline HIL & Hardware in loop \\
WR & Waveform relaxation \\
VLSI & Very large scale integrated \\
HVDC & High voltage DC \\
PLL & Phase locked loop \\
RTAI & Real time application interface \\
TCSC & Thyristor controlled series capacitor \\
\hline
\end{tabular}

While proof of concept simulations have been performed (Kulkarni et al 2010), practical testing is required to bring out the feasibility of the scheme. Towards this end, this paper presents results of experiments using actual implementation of real-time controllers, interacting with simulated models of power apparatus through real-time play-back. We present two case studies, which are briefly described below.

\subsection{HVDC Controller testing}

We perform the following experiments: (a) A non-real-time digital simulation of a detailed model of a HVDC power apparatus and its controller. (b) Waveform relaxation based testing using a non-real-time simulator for the HVDC power apparatus and a real-time digital implementation of the controller. (c) Application of the same digital controller to a real-life, but scaled laboratory implementation of the HVDC power apparatus.

\subsection{TCSC Damping controller testing}

We have performed experiments (a) and (b) given above, except that a low frequency model of a power system and a TCSC is used instead of the HVDC system.

The aim of these experiments was to bring out any practical issues and difficulties relating to the implementation of the WR based iterative testing scheme, as well as to validate the results obtained from the converged solution in the WR scheme. A detailed description of the experiments and the results are presented in the following sections. Table 1 lists acronyms used in this paper.

\section{WR based testing of a HVDC controller}

Three sets of experiments are designed as a part of the evaluation of the WR scheme. The details and intent of the the experiments are described below.

Experiment I: Model the HVDC Power Apparatus and the Controller which will be used for WR testing, in a non-real time power system simulator (SimPowerSystems Toolbox of MATLAB®is used). A closed loop simulation of the coupled system is carried out - see figure 4.

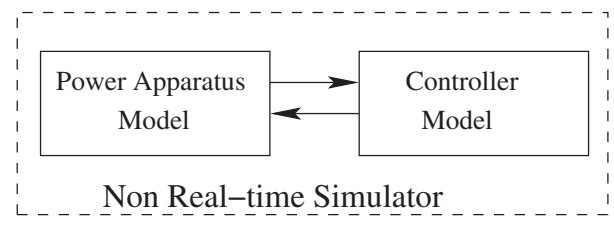

Figure 4. Experiment I: Simulated closed loop behaviour. 


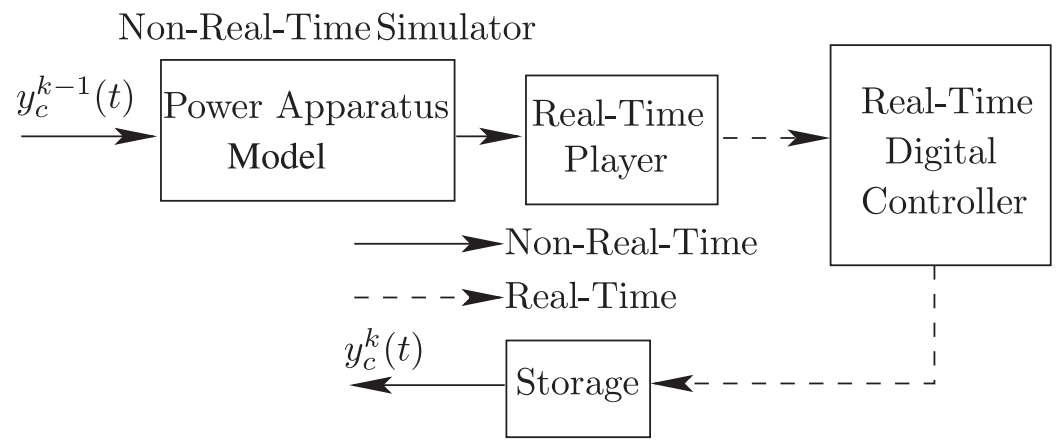

Figure 5. Experiment II: WR based iterative controller testing.

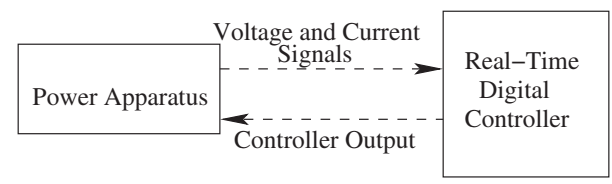

Figure 6. Experiment III: Controller interfaced with real-life power apparatus.

Experiment II: Model the HVDC Power Apparatus in the non-real time power system simulator as in Experiment I. However, this simulation is interfaced with a real-time implementation of the controller through real-time playback and storage - see figure 5. Iterations are performed as per the WR method, till convergence is attained.

Experiment III: The real-life, but scaled laboratory implementation of the HVDC power apparatus (a simulation model of which was used in the Experiments I and II) is interfaced with the real-time implementation of the controller used in Part II - see figure 6. The results of Experiment I, II and III are compared for a similar set of disturbances and set-point changes.

\section{Remark:}

(i) The aim of Experiment II was to check the practical feasibility of the WR controller testing scheme. This is the main experiment; it is the way WR method will be used in practice for controller testing. The aim of the experiments was not to evaluate the controller performance per se, but to check whether there were any practical issues with iterative application/playback/storage and automating the process, whether the WR method converged, and whether the WR scheme correctly captured the closed loop controller response when it converged.

(ii) The comparison of the results ${ }^{1}$ of the three experiments for a similar set of disturbances, serves as a sanity check on the WR results. Ideally, the results should match exactly, although this will not be true in practice. The extent of the match between the results of the

\footnotetext{
${ }^{1}$ Note that in Experiment II, by 'results' we refer to the waveforms obtained after the WR iterations have converged.
} 
three will depend on the fidelity of the controller and apparatus models used in Experiment I and the power apparatus model used in Experiment II.

(iii) The Experiments I, II and III mimic the controller design phase, controller testing phase and field testing stage, respectively of a power system controller.

\subsection{The HVDC system and its controller}

2.1a HVDC power apparatus: The laboratory set-up of the HVDC system to be used in Experiment III is shown in figure 7. The three phase AC supply is at $230 \mathrm{~V}$ (rms, line-to-line), and is obtained by stepping down the laboratory supply.

The rectifier and inverter are six pulse line-commutated thyristor bridges. At the ac side of the rectifier, a bank of three single phase transformers $(230 / 50 \mathrm{~V})$ connected in star on both the primary and the secondary side are used. A similar set-up exists at the inverter terminals. The transformers are provided with taps, with the tap on the inverter side set lower than that at the rectifier side. The dc link consists of a smoothing inductor. The parameters of this system are given in table 2 .

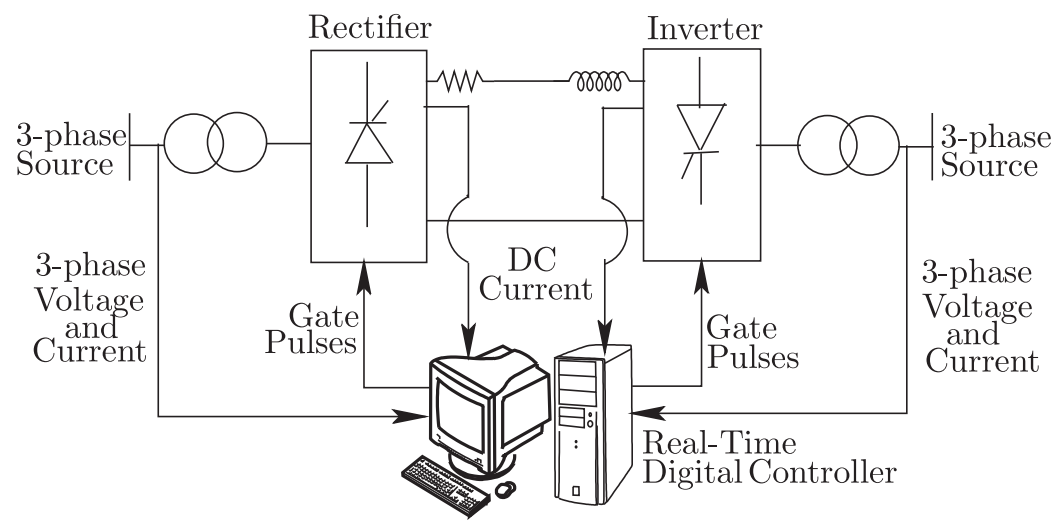

Figure 7. Laboratory HVDC set-up.

Table 2. HVDC apparatus details.

\begin{tabular}{|c|c|c|}
\hline \multirow{3}{*}{ a) } & \multicolumn{2}{|c|}{ Converters: 3-phase, fully controlled 6-pulse } \\
\hline & Input voltage & $50 \mathrm{~V}$ \\
\hline & Thyristors Make & ST TYN612M \\
\hline \multirow[t]{4}{*}{ b) } & DC Link & \\
\hline & Resistance & $4 \Omega$ \\
\hline & Inductance & $360 \mathrm{mH}$ \\
\hline & Inductor rating & $5 \mathrm{~A}$ \\
\hline \multirow[t]{3}{*}{ c) } & Star-Star bank of 3 single-pha & \\
\hline & Voltage Rating (line-to-line) & $230 / 50 \mathrm{~V}$ \\
\hline & Secondary Current Rating & $5 \mathrm{~A}$ \\
\hline \multirow[t]{2}{*}{ d) } & Controller details & \\
\hline & Time step (h) & $60 \mu \mathrm{s}$ \\
\hline
\end{tabular}


The system is modelled for Experiments I and II using using the available library elements for thyristors, passive electrical elements, and logical/control blocks of the SimPowerSystems Tool-

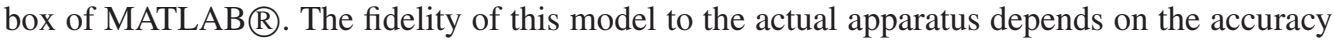
of the apparatus parameters (these are either estimated or obtained from data sheets). For Experiment I, the non-real-time closed loop simulation was carried out with Runge-Kutta $4^{\text {th }}$ order method with a fixed time step of $60 \mu$ s. Similarly, the non-real-time simulation of the power apparatus model in Experiment II was carried out with Runge-Kutta 4th order method with a fixed time step of $60 \mu \mathrm{s}$.

2.1b HVDC controller: The basic function of the controller is to regulate the current at its setpoint. The current in the dc link is controlled by adjusting the firing angles of the two converters. The synchronization is provided by a Phase-Locked Loop (PLL) which locks on to the ac voltages at the primary of the transformers at both converter ends. Startup is achieved by giving the switch-on signals to a pair of thyristors of one leg of the six-pulse inverter. Thereafter, pulses are given to the rectifier thyristors in the usual six-pulse bridge sequence, the delay angle being controlled by the current regulator. Once the current in the link is set-up, the inverter is fired in the constant firing angle delay $\left(>90^{\circ}\right)$ mode, with a conservative margin to prevent commutation failure. If the delay angle at the rectifier reaches its lower limit, the current control shifts to the inverter, with the current order reduced by a small margin. Power reversal function is achieved by ramping down the firing angle delay of the inverter to $15^{\circ}$, while current is still regulated by the converter at the other end. This changes the polarities of the DC side voltages at both ends. The schematic of the controller is shown in figure 8 .

For Experiments II and III, the HVDC controller is implemented on a desktop computer using Real Time Application Interface (RTAI)(RTAI 2000) working on a Linux operating system. RTAI is a real-time extension for the Linux kernel which allows us to write applications with strict timing constraints ('hard real-time'). RTAI provides the ability to make Linux fully preemptable. It offers the services of the Linux kernel core (device drivers, networking, etc.), adding

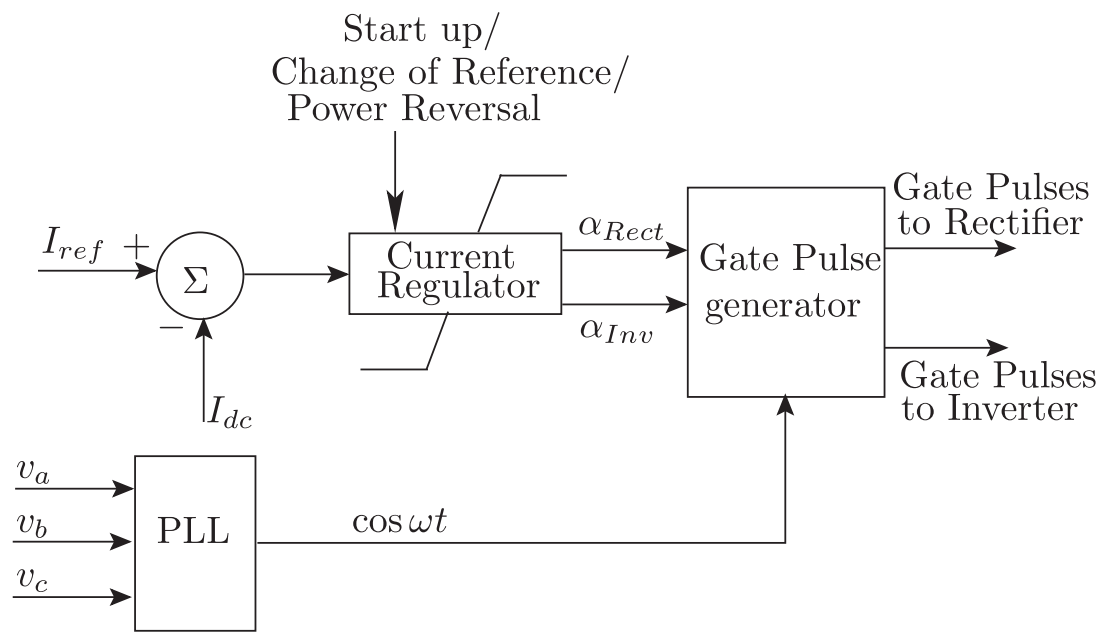

Figure 8. Controller schematic. 
Table 3. PCI card details.

\begin{tabular}{lll}
\hline & Make & Advantech $P C I-1710 \mathrm{HG}$ \\
Analog input : & \\
& Voltage range (used) & $0 \mathrm{~V} \sim 10 \mathrm{~V}$ \\
& Channels & 12 \\
& ADC resolution & $12 \mathrm{bit}$ \\
& ADC sampling rate & $100 \mathrm{kHz}$ \\
b) & Analog output : & 2 \\
& Channels & $12 \mathrm{bit}$ \\
c) & Digitation input : & 16 \\
& Channels & $0.4 \mathrm{~V} \sim 2.4 \mathrm{~V}$ \\
Voltage range & Digital output : & 16 \\
& Channels & $0.4 \mathrm{~V} \sim 2.4 \mathrm{~V}$ \\
\hline
\end{tabular}
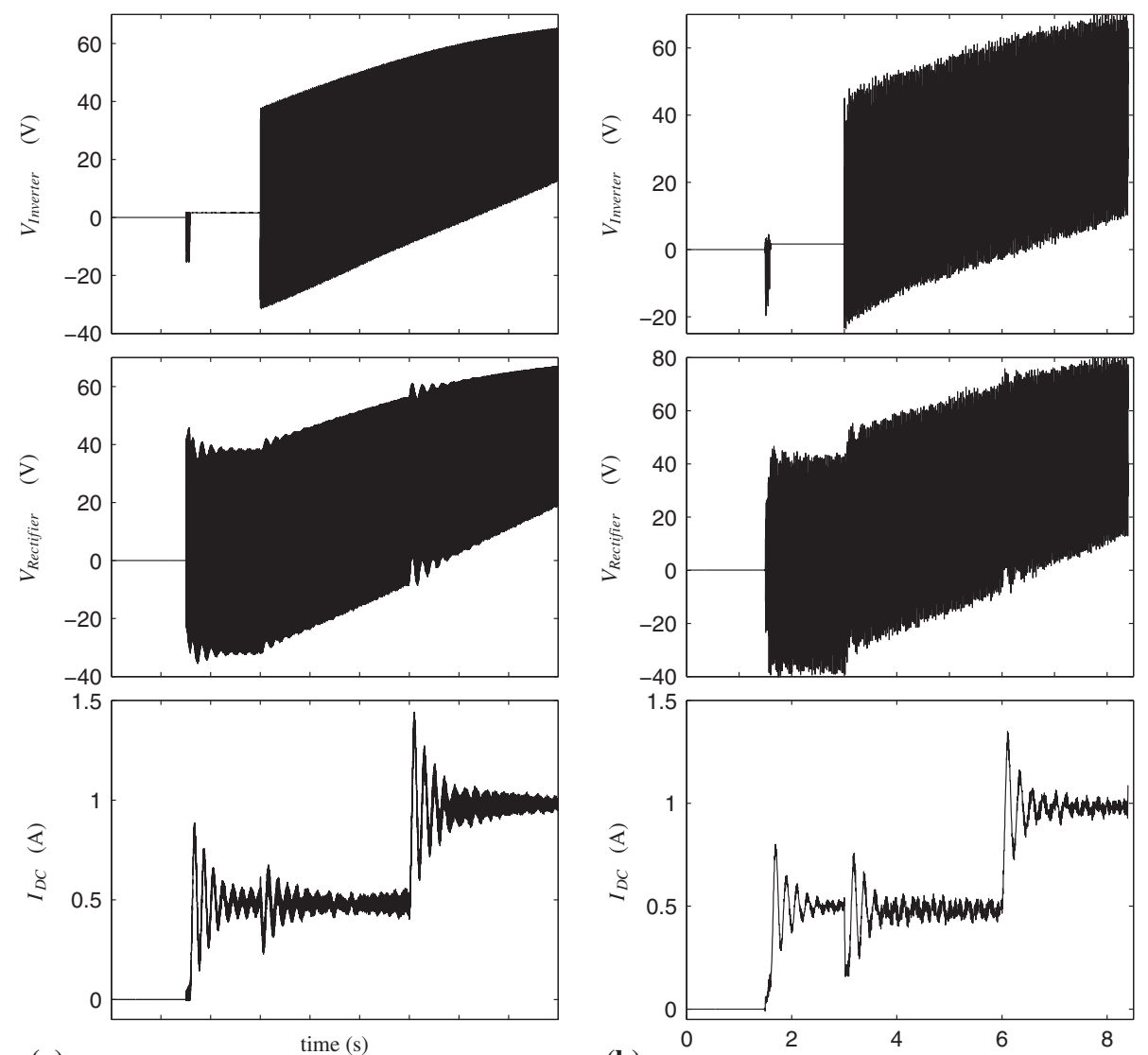

(a)

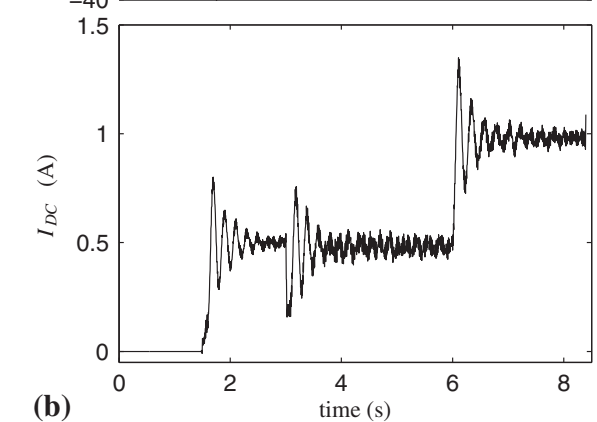

Figure 9. Startup and step increase in reference current (a) Non-real-time simulation of apparatus and controller (Experiment I) and (b) Converged waveforms of WR iterations with a Non-real-time simulator and Real-time controller implementation (Experiment II). 
to it the features of an industrial real-time operating system. For example, the implementation of a digital controller using RTAI, see (Meghawani \& Kulkarni 2008).

The controller requires the measured values of DC link current, and the AC side voltages of the rectifier and the inverter, for which a measurement card is fabricated. A pulse transformer based circuit provides isolation and conveys the train of firing pulses from the controller to the thyristors.

The current and voltage measurements from the measurement card are given to the computer through a data acquisition card (see table 3). The analog data from the HVDC Link is not read by the controller simultaneously. The controller reads one analog input channel every $60 \mu \mathrm{s}$.

For Experiment I, a simulation model of the controller is used. While the controller functionalities described above are modelled, details of analog to digital conversion, numerical precision of measurements, model of the measurement card and firing angle circuit are not modelled in the simulation program.

\subsection{Results}

We present the responses obtained from the three Experiments under the following situations:

(i) Startup of DC link and a subsequent step increase in the reference current at $6 \mathrm{~s}$.

(ii) Startup of DC link, a subsequent power flow reversal at $4 \mathrm{~s}$.

(iii) Startup of DC link but with a missing gate pulse to one thyristor of the rectifier bridge. The aim of this is to check the performance of the testing scheme when there is a gross problem in the controller.

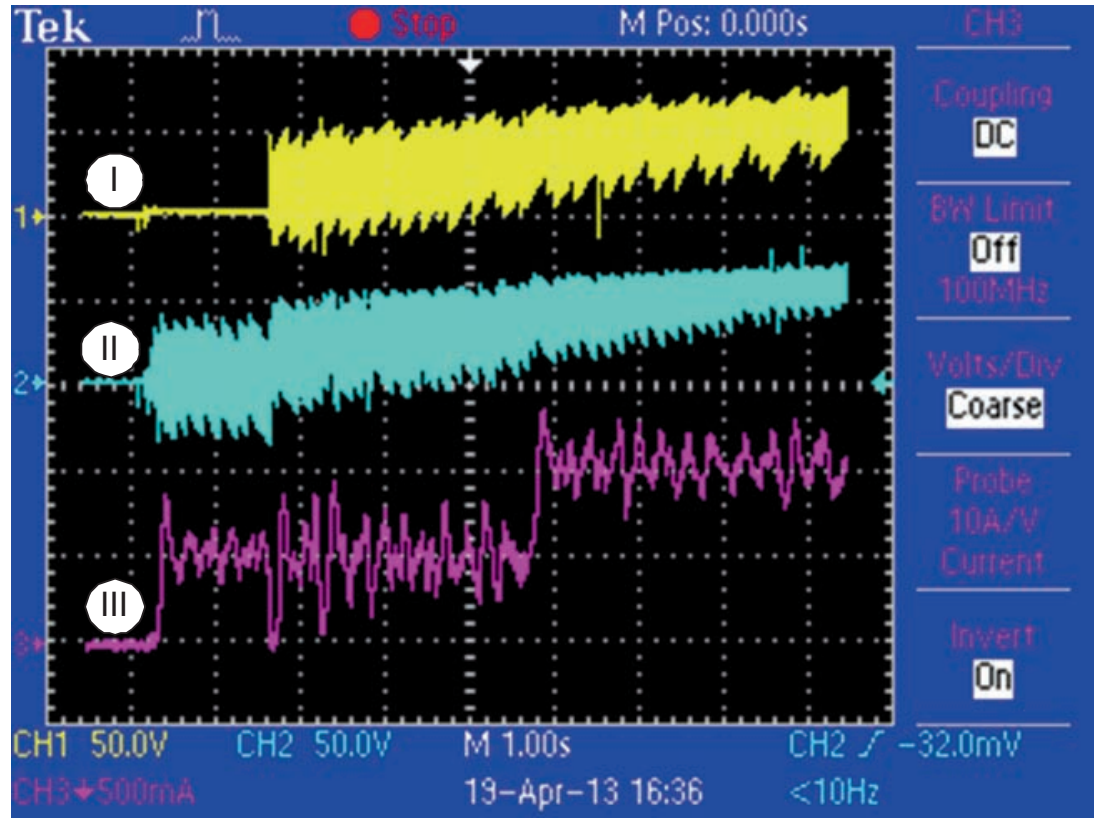

I. Inverter DC Voltage, II. Rectifier DC Voltage, III. DC Link Current

Figure 10. Start-up and step increase in reference current: Laboratory power apparatus and Real-time controller implementation (Experiment III). 
2.2a Starting of the HVDC link and step increase in reference current: The results of Experiment I and II are shown in figure 9, while the results of Experiments III are shown in figure 10. Note that from time $t=0 \mathrm{~s}$ to $t=1: 5 \mathrm{~s}$ no converter is provided with firing pulses. At time $t=$ $1.5 \mathrm{~s}$, the rectifier thyristors are triggered and at the same time the two thyristors of one leg of the inverter are provided continuous gate pulses. This establishes current in the DC link which is regulated by the current regulator by controlling the firing delay angle of the rectifier. The current reference at startup is $0.5 \mathrm{~A}$. Regular switching pulses are delivered to the inverter at time $t=3 \mathrm{~s}$ with an initial delay angle of $90^{\circ}$ which is ramped up towards $150^{\circ}$ at a slow rate. The inverter works with a constant firing delay of $150^{\circ}$ thereafter.

A step change in reference current from $0.5 \mathrm{~A}$ to $1.0 \mathrm{~A}$ is given at $t=6.0 \mathrm{~s}$. The results shown in the figures 9 and 10 show a good match. In Experiment II, it takes 240 iterations for the WR method to converge.

2.2b Starting of the HVDC link and power reversal: The power reversal order is given at a reference current of $0.5 \mathrm{~A}$ at $t=4.2 \mathrm{~s}$. The startup is done as before. The firing angle of the
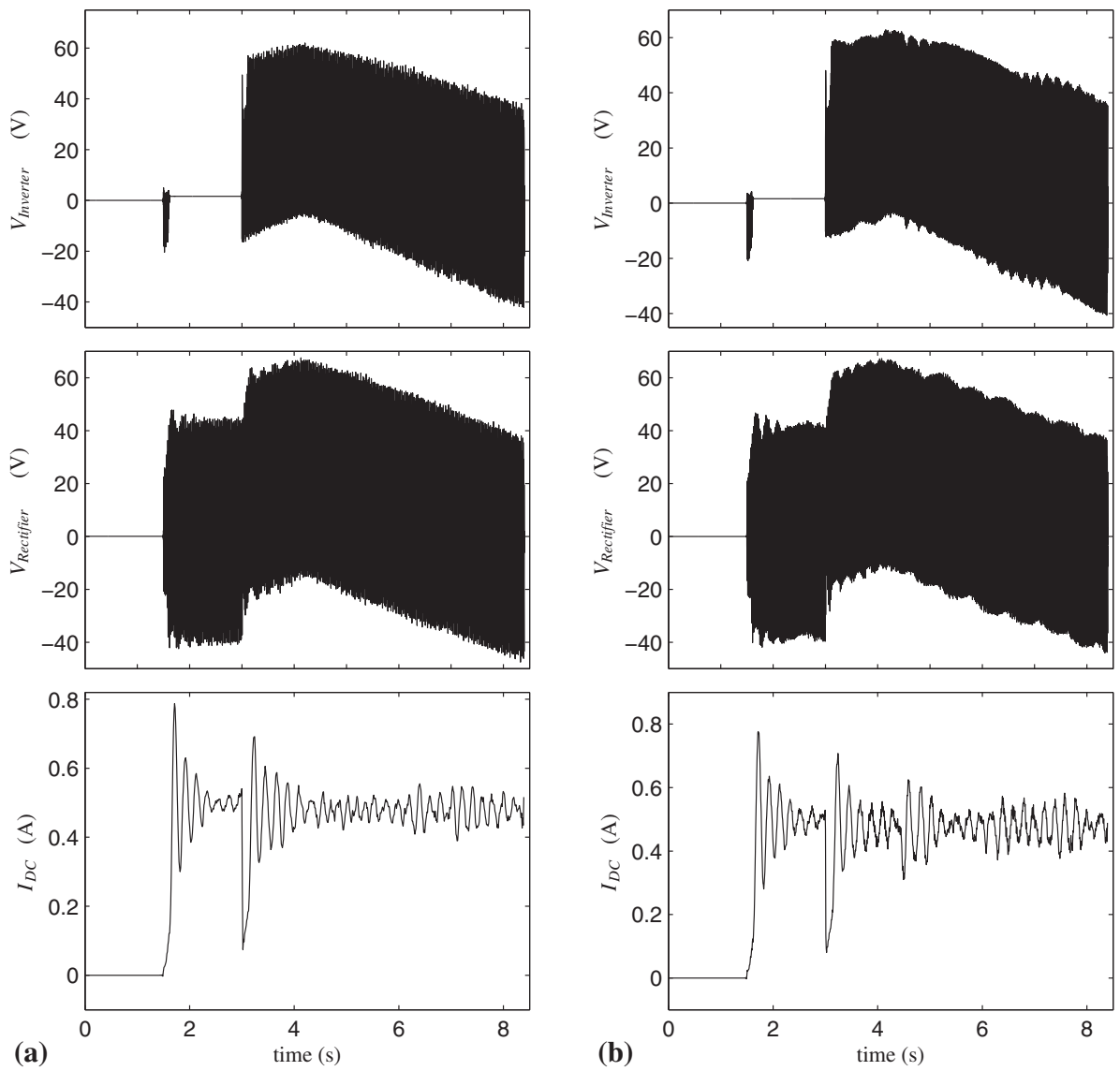

Figure 11. Start-up and power reversal: (a) Non-real-time simulation of apparatus and controller (Experiment I) and (b) Converged waveforms of WR iterations with a Non-real-time simulator and Real-time controller implementation (Experiment II). 
'inverter' is ramped down to a value of $15^{\circ}$, while current is still regulated by the 'rectifier', which results in reversal of roles between the converters. The responses are given in figures 11 and 12. During the process of reversal, the polarity of dc link voltages at both ends changes from a positive value to negative value while current direction remains the same. Convergence of the WR method is obtained in 245 iterations.

2.2c Gate pulse to one thyristor missing: This case is similar to the first one except that the Gate Pulse to one thyristor in the rectifier was blocked. The aim of this study is to check whether the WR method converges under these conditions (a faulty controller). This is indeed so; convergence is attained in 195 iterations. The responses are shown in figures 13 and 14 .

\subsection{Discussion}

(i) The final results of the three experiments did not bring out any unwelcome surprises; the WR method did converge and the converged responses matched fairly well with those obtained from Experiments I and III. There were some differences which might be attributed to modelling, and errors in estimated parameter values of the apparatus. The laboratory power supply was also not perfectly sinusoidal, as was assumed in the models of Experiment I and II.

(ii) In Experiment II, all iterations always included the start-up process, and therefore controller is reset to its original state at the beginning of each iteration. A potential difficulty exists if there is no easy access/control of the controller states. Most non-real-time simulators have a snapshot facility which allows us to set initial conditions, but this may be an issue with a

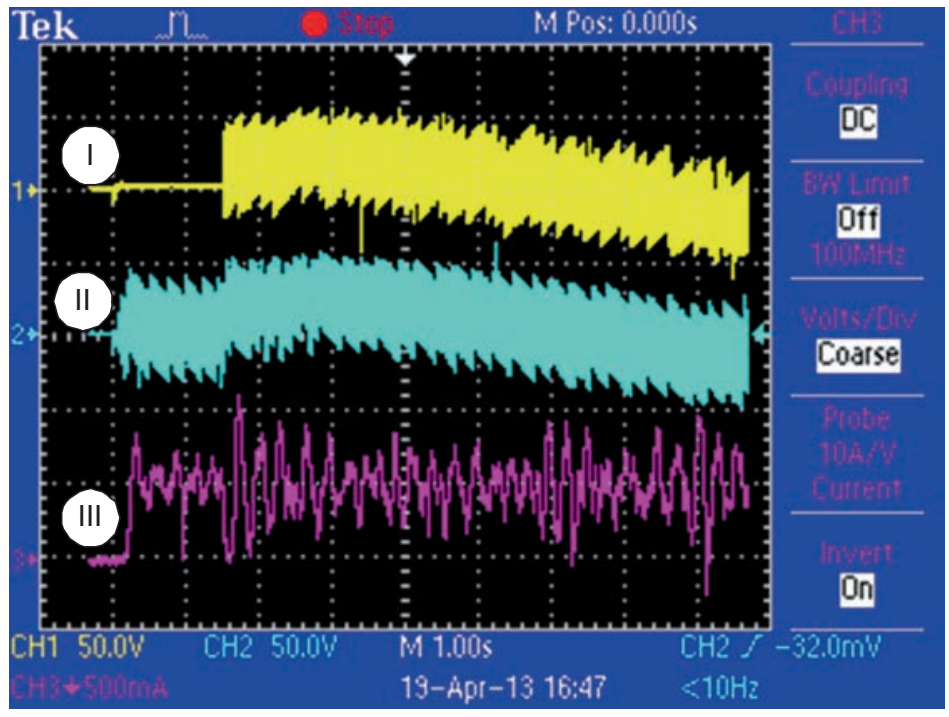

I. Inverter DC Voltage, II. Rectifier DC Voltage, III. DC Link Current

Figure 12. Start-up and Power reversal: Laboratory power apparatus and Real-time controller implementation (Experiment III). 

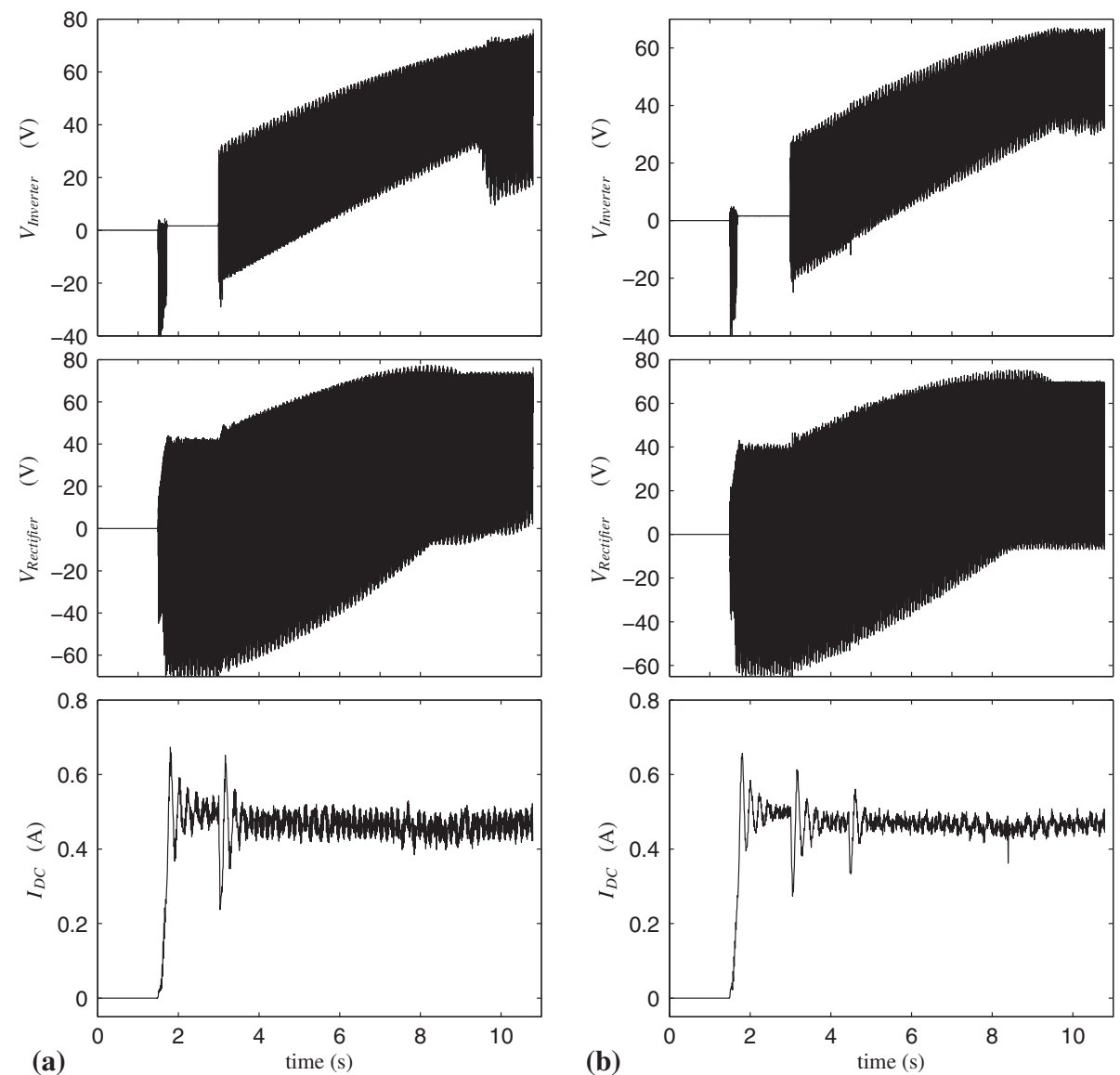

Figure 13. Start-up and step change in current reference, with pulse to one rectifier thyristor missing: (a) Non-real-time simulation of apparatus and controller (Experiment I) and (b) Converged waveforms of WR iterations with a Non-real-time simulator and Real-time controller implementation (Experiment II).

real-time controller especially if it available as a black-box from a manufacturer. Ability to start or bring the controller to the desired state is required if an improvisation like windowing is to be implemented improve convergence time.

\section{TCSC damping controller}

We now study the performance of the WR method for a Single Machine Infinite Bus (SMIB) system with a Thyristor Controlled Series Compensator which has a modulation controller for damping of power swings. We perform experiments similar to Experiments I and II of the previous section (see figures 4 and 5). The power system including the TCSC is simulated in nonreal-time using a low frequency model of the system (a transient stability model). The system is shown in figure 15 and the parameters of the system are given in table 4. 


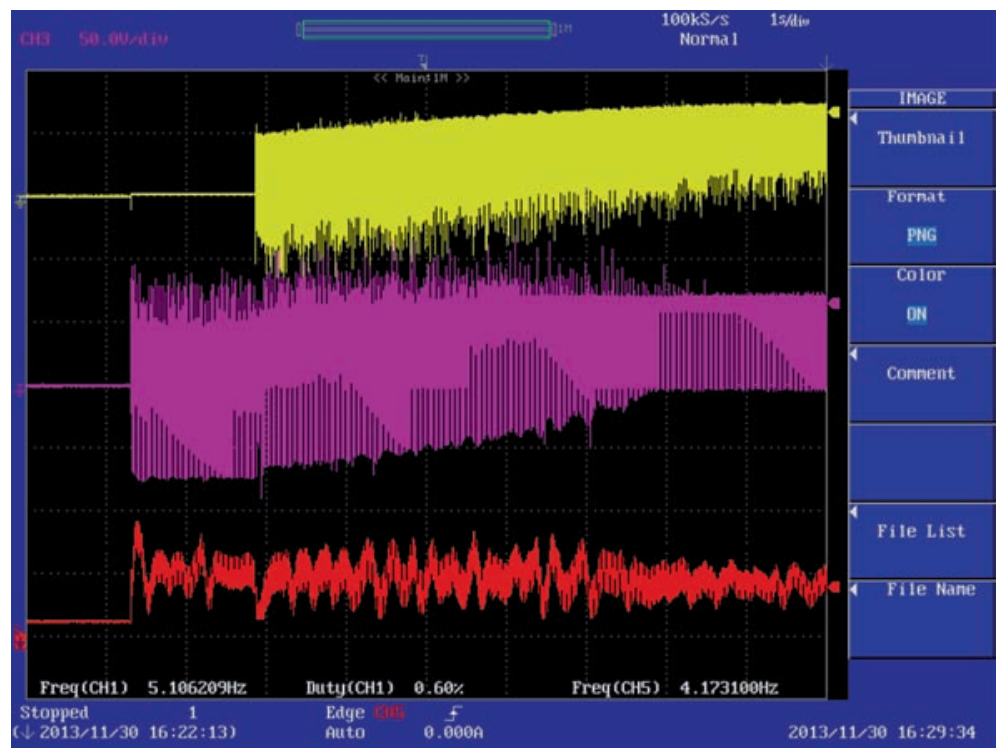

I. Inverter DC Voltage, II. Rectifier DC Voltage, III. DC Link Current

Figure 14. Start-up and step change in current reference, with pulse to one rectifier thyristor missing: Laboratory power apparatus and Real-time controller implementation (Experiment III).

As in the HVDC case, we use an RTAI implementation of the controller for the WR based testing scheme. The non-real-time simulation is carried out using the SIMULINK Toolbox of MATLAB. We first present the results of a closed loop simulation including the controller, using the non-real time simulator, as in figure 4 for a three-phase fault on one of the two lines near the terminals of the generator, which is cleared by tripping out the faulted line. The response without the damping controller is poorly damped, but with a damping controller we obtain good damping as shown in figure 16 a.

The results obtained using WR iterations are shown in figure 16b. The converged waveform matches well with the one shown in figure 16a. The number of iterations till convergence is small compared to the HVDC example. This is not surprising, considering the relative complexity of the HVDC system, which involves fast power electronic switchings and complex control.
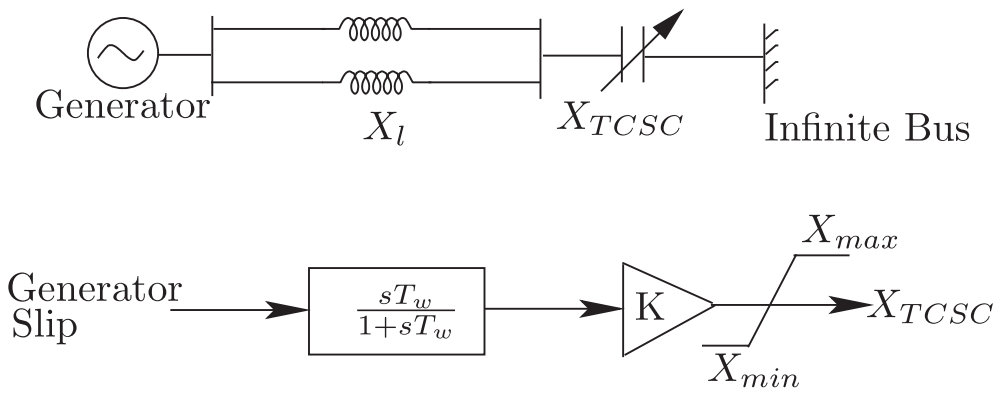

Figure 15. SMIB system with a TCSC, and the TCSC damping controller. 
Table 4. System parameters for SMIB system with TCSC.

\begin{tabular}{lll}
\hline & Item & Value \\
a) & Generator (Classical Model) & \\
& Reactance $x^{\prime} d$ & $0.1 \mathrm{pu}$ \\
& Inertia constant $\mathrm{H}$ & $2 \mathrm{~s}$ \\
& Base speed $\omega_{b}$ & $314.15 \mathrm{rad} / \mathrm{s}$ \\
b) & \\
& Lines : & $0.18 \mathrm{pu}$ \\
cquivalent reactance $X_{l}$ & \\
& TCSC : & $0.02 \mathrm{pu} / \mathrm{rad} / \mathrm{s}$ \\
& Nominal Gain $K$ & $10 \mathrm{~s}$ \\
& Washout time constant $T w$ & $0.01 \mathrm{pu}$ \\
& $X_{\min }$ & $0.04 \mathrm{pu}$ \\
& $X_{X \max }$ & \\
d) & $100 \mathrm{~ms}$ \\
& Fault clearing time & $1 \mathrm{~ms}$ \\
\hline
\end{tabular}
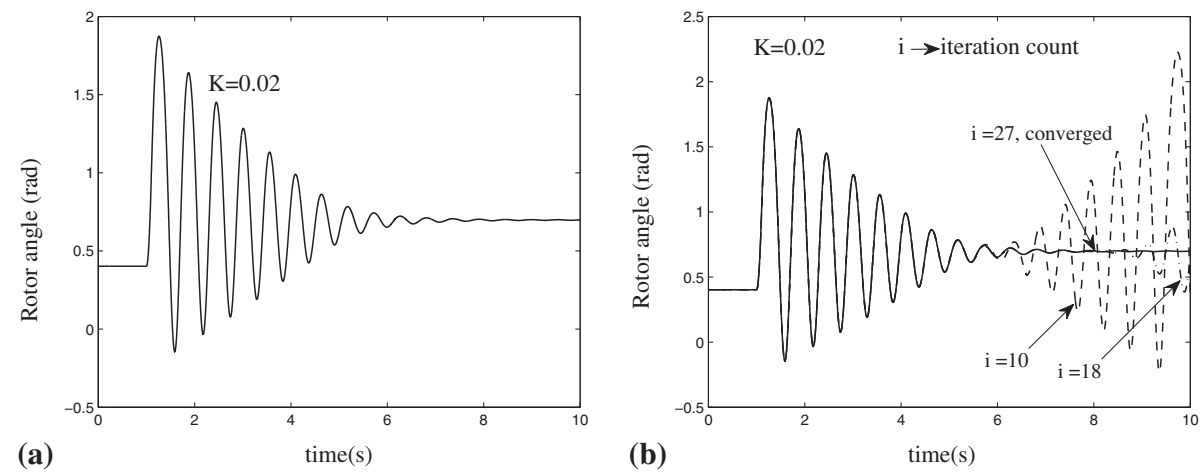

Figure 16. Results of (a) closed loop non-real time simulation (Experiment-I) and (b) WR based iterative playback testing (Experiment II). Note that the iterations converge.
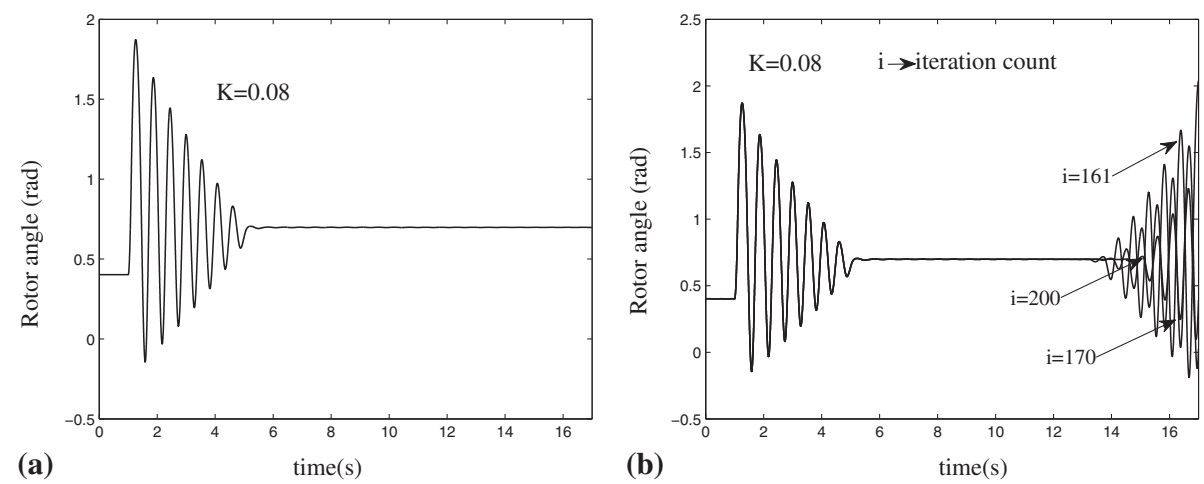

Figure 17. Results with higher gain: (a) closed-loop non-real time simulation (Experiment-I) and (b) WR based iterative playback testing (Experiment II). Note that the iterations do not converge. 


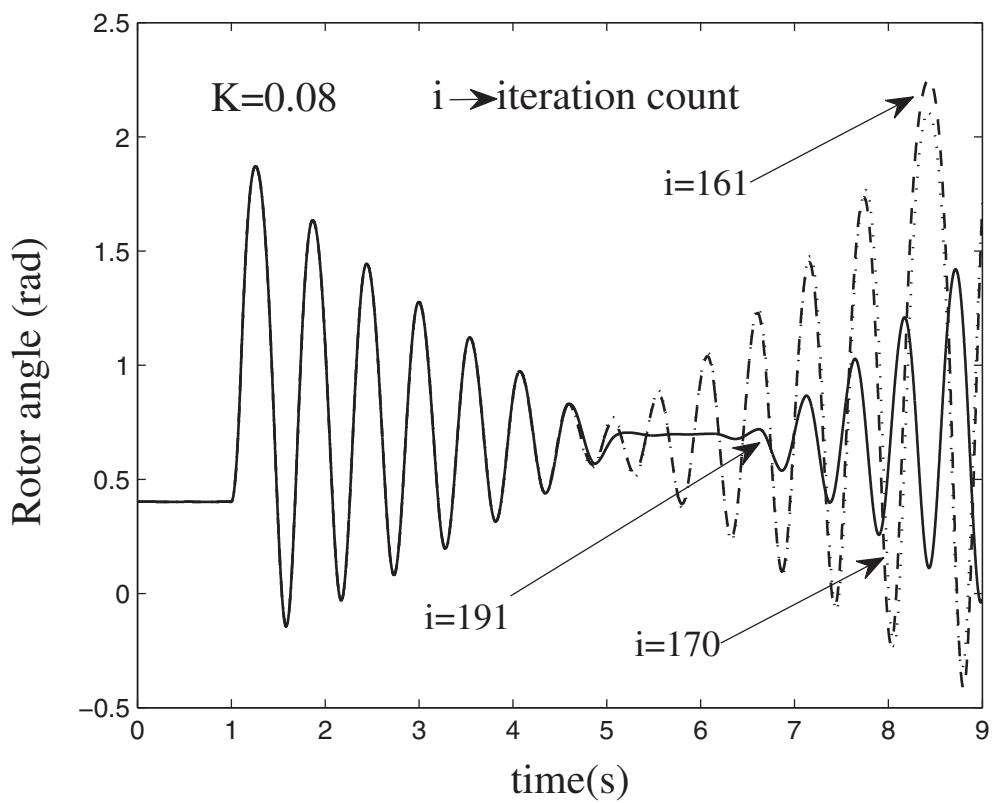

Figure 18. WR based iterative playback scheme (Experiment II) with high controller gain and reduced time duration. Note that the iterations do not converge.

\subsection{Convergence problem at higher gain}

We now present the case where the gain value is increased to $0.08 \mathrm{pu} / \mathrm{rad} / \mathrm{s}$ from the nominal value $0.02 \mathrm{pu} / \mathrm{rad} / \mathrm{s}$. The result from the closed loop non-real-time simulation is shown in figure 17a, while the results of the WR scheme are shown in figure 17b.

A convergence problem in the WR scheme is visible near the end of interval; the iterations do not converge even the number of iterations is increased to 200. Even when the time interval for simulation is reduced to half, the convergence problem persists as seen in figure 18.

To diagnose the convergence problem, we carry out a simulation of the WR iterations using a non-real-time simulator, i.e. the play-back and controller is also simulated using a non-real-time

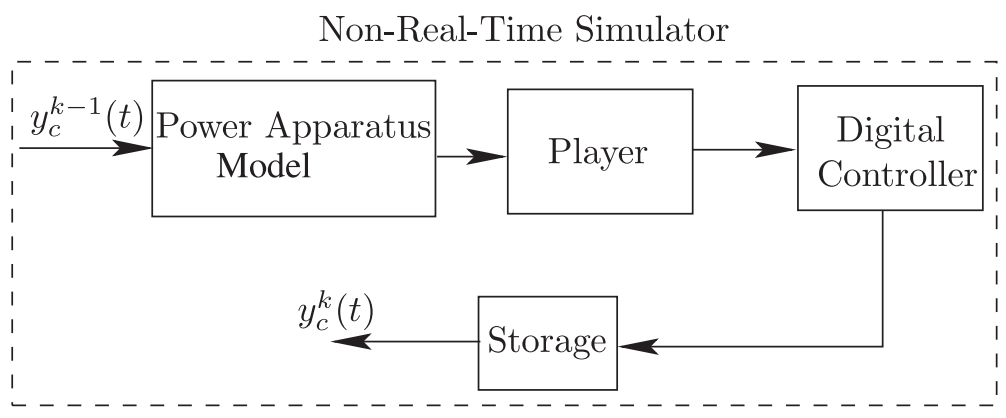

Figure 19. WR based iterative scheme simulated using a non-real-time simulator. Contrast this with figure 5 . 


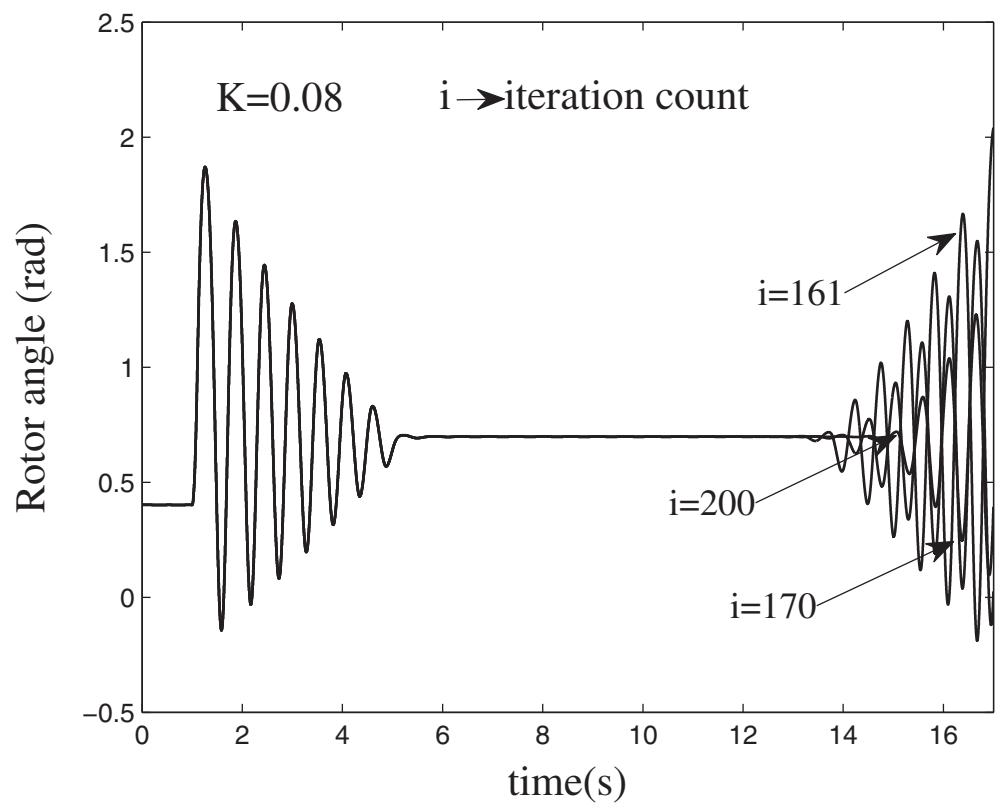

Figure 20. Simulated WR scheme as in figure 19, which includes the modelling of lower numerical precision in controller and play-back.

simulator as shown in figure 19. Our observations using this simulated WR environment are as follows.

(i) The WR iterations in the non-real-time environment converge to the same solution as in figure $17 \mathrm{a}$, if the numerical precision of the controller output is the default precision of the non-real- time simulator (double precision).

(ii) If 12 bit numerical precision of the controller output is modelled, as present in the real-time implementation of the controller, non-convergence is seen at the higher gain (see figure 20), which is similar to what is observed in figure $17 \mathrm{~b}$.

(iii) If the gain value is low, the WR iterations converge even with the lower precision controller output. This replicates what is seen in figures $16 \mathrm{~b}$.

(iv) If the higher gain in the controller is used along with a first order low pass filter $1 / 1+0.02 s$, then it is observed that the WR converge with the lower precision controller output.

Therefore, we that WR conclude convergence may be hampered if the controller has a high transient gain and uses low numerical precision. It should be noted in the closed loop case (e.g., HIL simulation), numerical round-off would generally have a small effect on the response, and the errors would not 'accumulate' as there are no iterations involved.

However, in the WR based scheme, the round-off noise is applied at every iteration and also changes at every iteration. This has an impact on the convergence itself. The round-off due to precision change may be modelled as iteration dependent noise, as shown in figure 21 . The theoretical behaviour of WR iterations under this situation, and the ways to avoid the convergence problem are being investigated by the authors. 

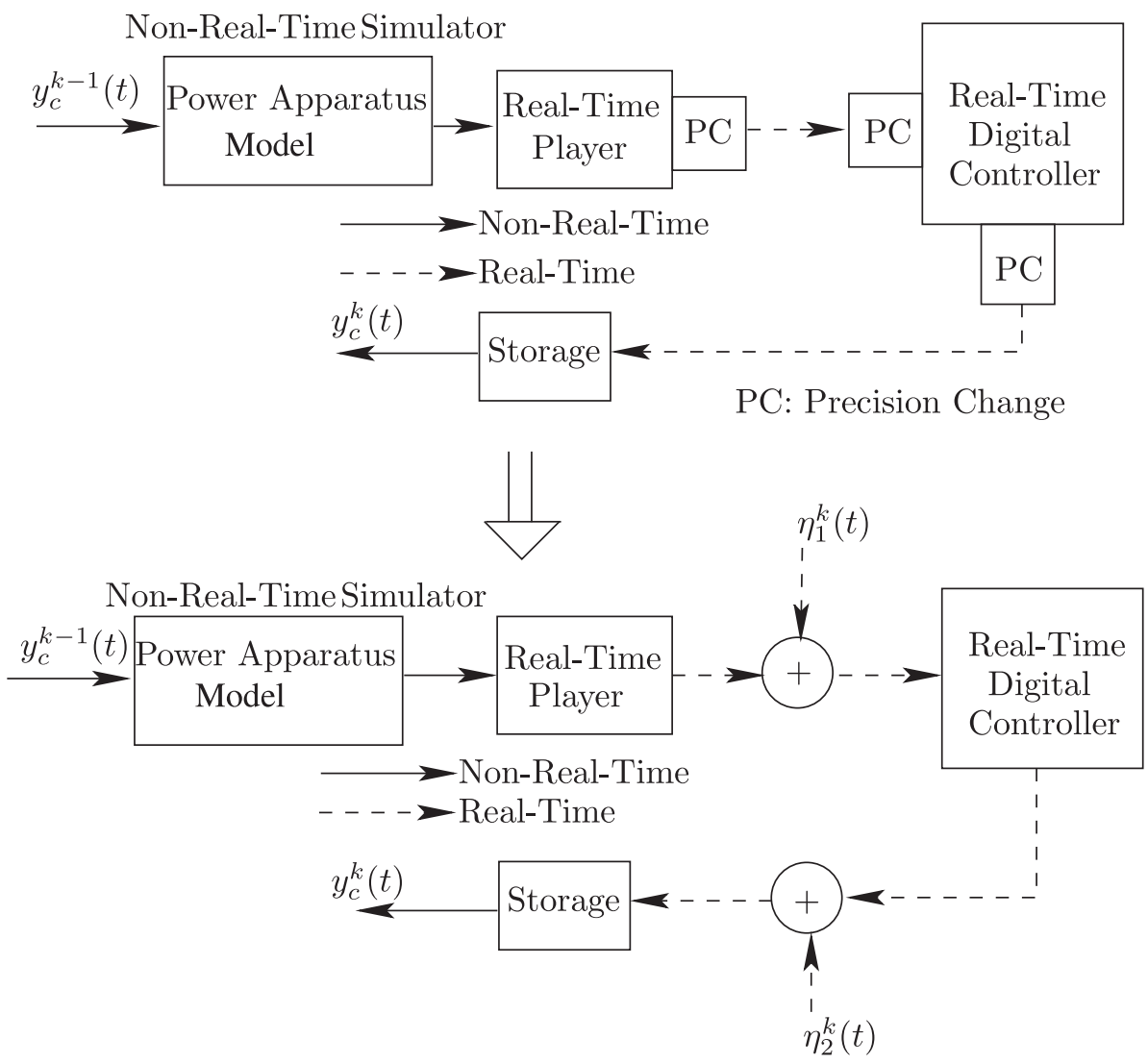

$\eta_{1}^{k}(t)$ and $\eta_{2}^{k}(t)$ are iteration dependent round-off noise

Figure 21. Modelling of precision errors as iteration-dependent noise.

\section{Conclusion}

The experiments carried out using the WR scheme and its comparison with closed-loop simulations and real-life behaviour with scaled power apparatus, show that this is a viable method for controller testing. The experiments give us the necessary confidence to utilize the method for realistic controller testing, but also flag two important practical issues.

(i) To implement schemes like windowing to speed-up the overall convergence time, it would be necessary to ensure that the controller be directly ready at the desired initial conditions of its states, without requiring a complete resetting of the states and a start-up at every iteration. The means to facilitate this need to be investigated, especially if the controller is available as a black-box from a manufacturer, without complete access to all the internal state variables.

(ii) Convergence problems due to iteration-dependent noise due to the use of lower numerical precision in the controller/play-back, especially when the controller has a high transient gain. This problem is system-dependent and not generic. Theoretical studies and practical improvisations are required to overcome this problem, if it is encountered. 
These issues do not detract from the viability of the WR based scheme as a low-cost alternative to HIL based controller testing, and are interesting topics for further research and development.

\section{References}

Kezunovic M and McKenna M 1994 Real-time digital simulator for protective relay testing. IEEE Comput. Appl. Power 7(3): 30-35

Kezunovic M, Popovic T, Sevcik D and DoCarmo H 2003 Transient testing of protection relays: Results, methodology and tools. Int. Conf. Power Sys. Transients IPST, New Orleans, USA

Kulkarni A M, Salunkhe K, Chandorkar M C, Panda S P and Sankaranarayanan N 2010 Waveform relaxation based iterative real-time playback schemes for testing of wide area power system controllers. IEEE PES-GM, Minneapolis, USA:1-8

Lelarasmee E, Ruelhi A and Sangiovanni-Vintecelli A 1982 The Waveform relaxation method for time domain analysis of large scale integrated circuits. IEEE Trans. Comput. Aided Des. Integr. Circ. Sys. 1(3): 131-145

Meghwani A and Kulkarni A M 2008 Development of a laboratory model of SSSC using RTAI on Linux platform. Sadhana 33(5): 643-661

MATLAB and SIMULINK demos and documentation, [Online], Available: http://www.mathworks.com/ help/index.html

RTAI Programming Guide 1.02000 [Online], Available: http://www.rtai.org

Spong M Illic, Crow M L and Pai M A 1987 Transient stability simulation by waveform relaxation methods. IEEE Trans. Power Sys. 2(4): 943-949

White J, Sangiovanni-Vincentelli A, Odeh F and Ruehli A 1985 Waveform relaxation: Theory and practice. Trans. Soc. Comput. Simul. 2(1): 95-133

White J and Sangiovanni-Vintecelli A 1987 Relaxation techniques for the simulation of VLSI circuits. Boston: Kluwer Academic Publishers 